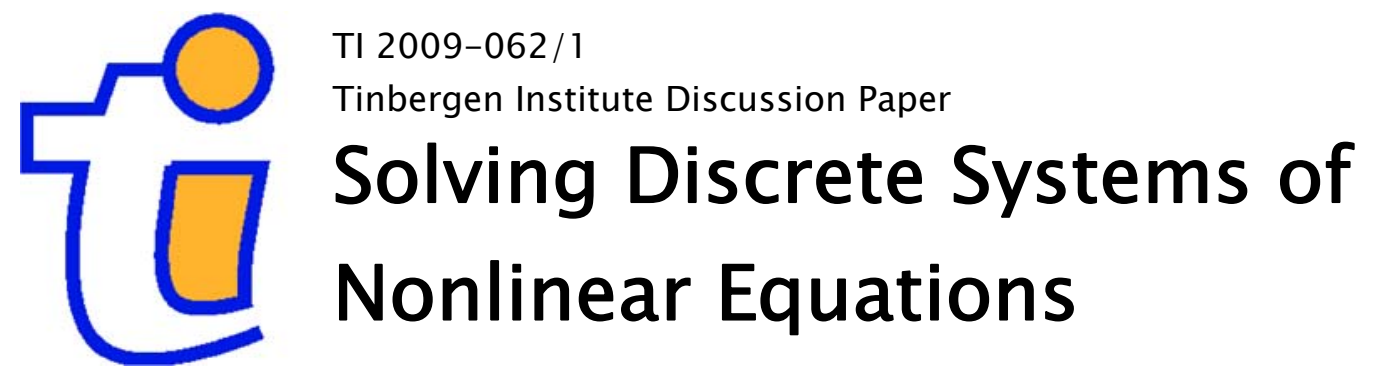

Gerard van der Laan'

Dolf Talman²

Zaifu Yang ${ }^{3}$

I VU University Amsterdam, Tinbergen Institute;

2 CentER, Tilburg University;

${ }^{3}$ Business Administration, Yokohama National University, Yokohama. 


\section{Tinbergen Institute}

The Tinbergen Institute is the institute for economic research of the Erasmus Universiteit Rotterdam, Universiteit van Amsterdam, and Vrije Universiteit Amsterdam.

Tinbergen Institute Amsterdam

Roetersstraat 31

1018 WB Amsterdam

The Netherlands

Tel.: +31(0)205513500

Fax: $+31(0) 205513555$

Tinbergen Institute Rotterdam

Burg. Oudlaan 50

3062 PA Rotterdam

The Netherlands

Tel.: + $31(0) 104088900$

Fax: $+31(0) 104089031$

Most TI discussion papers can be downloaded at http://www.tinbergen.nl. 


\title{
Solving Discrete Systems of Nonlinear Equations *
}

\author{
Gerard van der Laan ${ }^{\dagger}$, Dolf Talman ${ }^{\ddagger}$, Zaifu Yang ${ }^{\S}$
}

January 15, 2009

*This research was carried out while the third author was visiting CentER and the Department of Econometrics and Operations Research, Tilburg University, Tilburg. He gratefully acknowledges financial support by the Ministry of Education, Science and Technology of Japan, the Netherlands Organization for Scientific Research (NWO) and the CentER.

${ }^{\dagger}$ G. van der Laan, Department of Econometrics and Tinbergen Institute, Vrije Universiteit, De Boelelaan 1105, 1081 HV Amsterdam, The Netherlands, e-mail: glaan@feweb.vu.nl.

${ }^{\ddagger}$ A.J.J. Talman, CentER, Department of Econometrics \& Operations Research, Tilburg University, P.O. Box 90153, 5000 LE Tilburg, The Netherlands, e-mail: talman@uvt.nl.

$\S$ Z. Yang, Faculty of Business Administration, Yokohama National University, Yokohama 2408501, Japan. e-mail: yang@ynu.ac.jp. 


\begin{abstract}
In this paper we study the existence problem of a zero point of a function defined on a finite set of elements of the integer lattice $Z^{n}$ of the $n$-dimensional Euclidean space $\mathbb{R}^{n}$. It is assumed that the set is integrally convex, which implies that the convex hull of the set can be subdivided in simplices such that every vertex is an element of $Z^{n}$ and each simplex of the triangulation lies in an $n$-dimensional cube of size one. With respect to this triangulation we assume that the function satisfies some property that replaces continuity. Under this property and some boundary condition the function has a zero point. To prove this we use a simplicial algorithm that terminates with a zero point within a finite number of iterations. The standard technique of applying a fixed point theorem to a piecewise linear approximation cannot be applied, because the 'continuity property' is too weak to assure that a zero point of the piecewise linear approximation induces a zero point of the function itself. We apply the main existence result to prove the existence of a pure Cournot-Nash equilibrium in a Cournot oligopoly model. We further obtain a discrete analogue of the well-known Borsuk-Ulam theorem and a theorem for the existence of a solution for the discrete nonlinear complementarity problem.
\end{abstract}

Keywords: Discrete system of equations, triangulation, simplicial algorithm, fixed point, zero point.

AMS subject classifications: 47H10, 90C26, 90C33, 91B02. 


\section{Introduction}

Many problems arising from economics, engineering and mathematics can be formulated as a system of equations. The existence problem of a solution to a system of equations is called the zero point problem. This problem is associated with a function $f: X \rightarrow \mathbb{R}^{n}$ where $X$ is a subset of the $n$-dimensional Euclidean space $\mathbb{R}^{n}$. A point $x \in X$ is a zero point of $f$ if the image of $x$ is the origin, and $x$ is a fixed point of $f$ if $x$ is a zero point of the function $g$ given by $g(x)=f(x)-x$ and thus the image $f(x)$ of $x$ is $x$ itself.

Several fundamental theorems such as Brouwer fixed point theorem and Kakutani fixed point theorem concern the existence of fixed or zero point for the situation where the domain of interest is a convex and compact set in $\mathbb{R}^{n}$ and the mapping is continuous or upper semi-continuous. In his pioneering work Scarf $(1967,1973)$ gave the first constructive method for finding a fixed point on a compact convex set. Later on, more efficient simplicial algorithms for finding fixed or zero points were developed by Kuhn (1969), Eaves (1972), Merrill (1972), van der Laan and Talman (1979) among others.

Most of the existing fixed point theorems require that the domain be a convex set. This means that the components of the solution could be any real numbers. Such a solution can be a good approximation when the economic value associated with it is rather small. However, when the domain of interest involves significant indivisibilities such as houses, employees and equipments, the argument is no longer valid. Indeed, overwhelmingly many decision making variables of economic, business and engineering problems take only integer or rational numbers. For instance, essentially all commodities including divisible goods such as oil are traded in discrete quantities. Motivated by such practical consideration, we will investigate the zero point problem on a finite subset of the integer lattice $\mathbb{Z}^{n}$ in $\mathbb{R}^{n}$. This line of research can date back to Tarski (1955).

When the domain of interest is a discrete set, convexity and continuity have no direct meaning and have to be replaced by a kind of discrete version. In particular, convexity will be replaced by what is called integral convexity. The concept of integrally convex set was introduced by Favati and Tardella (1990) and has been well studied in the literature, see e.g. Murota (2003) and Fujishige (2005). Many well-known and important integral polyhedra generate integrally convex sets, for instance, the set $P \cap Z^{n}$ is integrally convex for any base polyhedron or generalized polymatriod $P$. The basic property of an integrally convex set is that its convex hull admits a simplicial subdivision or triangulation for which the set of vertices is equal to the set itself and every simplex is contained in a cube in $\mathbb{R}^{n}$ of size one.

In recent years several papers have appeared in which the existence of a discrete zero point was established for functions satisfying some property replacing continuity. Direction preserving functions are considered in Iimura (2003), Danilov and Koshevoy (2004), Iimura, 
Murota and Tamura (2005), and van der Laan, Talman and Yang (2006, 2007b). A function $f: \mathbb{Z}^{n} \rightarrow \mathbb{R}^{n}$ is direction preserving if for any two cell-connected points $x$ and $y$ in $\mathbb{Z}^{n}$, i.e., points within a same cube of size one, it holds that $f_{j}(x) f_{j}(y) \geq 0$ for every component $j=1, \ldots, n$. The more general class of locally gross direction preserving functions is studied in Yang $(2004,2008)$, Iimura and Yang (2005), van der Laan, Talman and Yang (2007a). A function $f: \mathbb{Z}^{n} \rightarrow \mathbb{R}^{n}$ is locally gross direction preserving if the inner product $f(x) \cdot f(y) \geq 0$ for any two cell-connected points $x$ and $y$ in $Z^{n}$. Talman and Yang (2009) showed existence of a zero point when $f$ is positive maximum component sign preserving, meaning that, for any two cell connected points $x$ and $y$ in $\mathbb{Z}^{n}, f_{j}(x)=\max _{h \in N} f_{h}(x)>0$ implies $f_{j}(y) \geq 0$. Although the class of positive maximum component sign preserving functions is also more general than the class of direction preserving functions, it is not comparable with the class of locally gross direction preserving functions in the sense that these two classes do not imply each other.

In this paper we consider a new class of functions with a property replacing continuity, namely the class of simplicially coordinatewise opposite extrema free (SCOEF) functions. A function $f$ on an integrally convex set is SCOEF if there exists a triangulation $T$ of the set such that for any two vertices $x$ and $y$ of any simplex in $T$ it holds that when some component of $f(x)$ has the highest absolute value and the same component of $f(y)$ has opposite sign, then the latter component is not the highest absolute value of $f(y)$. SCOEF functions further generalize positive maximum component sign preserving functions.

In most of the literature the existence of a zero (or fixed) point is shown by proving first that the piecewise linear approximation of the function on a simplicial subdivision has a zero point, and next that this zero point induces a zero point of the function itself. The existence of a zero point of the piecewise linear approximation can be proved either directly by using a fixed point argument such as Brouwer or Kakutani fixed point theorem, or constructively by applying a simplicial algorithm based on vector or integer labeling. Both the piecewise linear approximation approach and the vector labeling technique cannot, however, be applied to a SCOEF function, because the SCOEF function is so weak that the piecewise linear approximation of such a function may have a zero point, when the function itself does not have a zero point.

We adapt the integer labeling $2 n$-ray simplicial algorithm of van der Laan and Talman (1981) and Reiser (1981) and provide a constructive proof of the existence of a zero point of a SCOEF function from a finite integrally convex set in $\mathbb{R}^{n}$ to $\mathbb{R}^{n}$ satisfying some boundary condition. This boundary condition is similar to the ones that are used to guarantee the existence of a zero or fixed point of a continuous function on a compact convex set. Roughly, the condition states that on the boundary of the convex hull of the finite set, the function should point inwards. Starting at an arbitrary integral point of the set, the algorithm 
generates a unique sequence of adjacent simplices of variable dimension until a zero point is found. Since no simplex can be generated more than once and the number of simplices is finite because the set is finite, the algorithm terminates within a finite number of steps with a discrete zero point.

The existence result is applied to a discrete oligopoly model. In this model there is a finite number of firms, having quadratic individual cost functions. Firms produce a homogeneous good in discrete amounts and the inverse demand function is assumed to be linear. The equilibrium amounts are the fixed points of the reaction functions of the firms. Because the strategy set of every firm is finite, the discrete oligopoly model has an equilibrium in mixed strategies. We will show that the function whose components are the reaction functions of the firms is a SCOEF function with respect to some specific triangulation and satisfies the boundary condition. This will guarantee the existence of an equilibrium in pure strategies.

We also establish a discrete version of the well-known Borsuk-Ulam theorem on an arbitrary symmetric integrally convex set and a theorem for the existence of the discrete complementarity problem. In the latter problem a solution is a complementary point for a function that has the set of nonnegative integral vectors as domain. The SCOEF condition is slightly modified for this problem and the algorithm is adapted in order to find a complementarity point within a finite number of steps when the function satisfies the modified SCOEF condition and some (upper) boundary condition.

This paper is organized as follows. In Section 2 we introduce the class of simplicially coordinatewise opposite extrema free functions. In Section 3 we establish and prove the main existence result and apply this result to the discrete oligopoly model. In Section 4 we prove a discrete version of the Borsuk-Ulam theorem and discuss the discrete complementarity problem.

\section{Simplicially coordinatewise opposite extrema free functions}

For a given positive integer $n$, the set $\{1,2, \ldots, n\}$ is denoted by $N, \mathbb{R}^{n}$ is the $n$-dimensional Euclidean space, and $\mathbb{Z}^{n}$ is the integer lattice of $\mathbb{R}^{n}$. For $i \in N, e(i)$ denotes the $i$ th unit vector of $\mathbb{R}^{n}$ and $e(-i)$ denotes the $i$ th negative unit vector, i.e., $e(-i)=-e(i)$. Further $0^{n}$ denotes the zero vector. A set $X \subset \mathbb{R}^{n}$ is symmetric if $x \in X$ implies $-x \in X$. For an arbitrary set $X \subset \mathbb{R}^{n}, \bar{X}$ and $\partial X$ denote the convex hull of $X$ and the (relative) boundary of $X$, respectively.

Two points $x$ and $y$ in $Z^{n}$ are cell-connected if $\max _{i}\left|x_{i}-y_{i}\right| \leq 1$. The convex hull $\sigma\left(x^{1}, \ldots, x^{t+1}\right)$ of $t+1$ affinely independent points $x^{1}, \ldots, x^{t+1}$ in $\mathbb{R}^{n}$ is a $t$-simplex. $\mathrm{A}$ 
simplex is cubical if all its vertices belong to $\mathbb{Z}^{n}$ and every pair of two simplices is cellconnected. A subset $D$ of $Z^{n}$ is integrally convex if (i) $D=Z^{n} \cap \bar{D}$ and (ii) the set $\bar{D}$ is the union of a collection of cubical simplices. An integrally convex set $D$ in $Z^{n}$ is regular if $\bar{D}$ is $n$-dimensional and contains an integral point in its interior.

A collection $\mathcal{T}$ of $m$-simplices is a triangulation of an $m$-dimensional convex set $X$ in $\mathbb{R}^{n}$ if (i) $X$ is the union of all simplices in $\mathcal{T}$, (ii) the intersection of any two simplices in $\mathcal{T}$ is either empty or a common face of both, and (iii) any neighborhood of any point in $X$ only meets a finite number of simplices in $\mathcal{T}$. A triangulation is cubical if all its simplices are cubical. See Todd (1976) for more details on triangulations. A triangulation $\mathcal{T}$ of a symmetric convex set is symmetric if $\sigma \in \mathcal{T}$ implies $-\sigma \in \mathcal{T}$. It is known from Iimura, Murota and Tamura (2005) that for any integrally convex set $D$ there exists a cubical triangulation of its convex hull.

In the next section we prove that a function $f$ from an integrally convex set $D \subset \mathbb{Z}^{n}$ to $\mathbb{R}^{n}$ has a zero point when $f$ is simplicially coordinatewise opposite extrema free and satisfies some boundary condition. The condition concerns only those coordinates of the function having nonzero extreme value and states that there exists a triangulation of the convex hull of the domain such that within any simplex of the triangulation no coordinate of the function has values that are extreme in absolute value but are of opposite sign.

Definition 2.1 Let $D \subset \mathbb{Z}^{n}$ be an integrally convex set. A function $f: D \rightarrow \mathbb{R}^{n}$ is simplicially coordinatewise opposite extrema free (SCOEF) if there exists a cubical triangulation $\mathcal{T}$ of $\bar{D}$ such that for every simplex $\sigma$ of $\mathcal{T}$ and every two vertices $x$ and $y$ of $\sigma$ with $f(x) \neq 0^{n}$ and $f(y) \neq 0^{n}$, there exists no $k \in N$ such that

$$
f_{k}(x) f_{k}(y)=-\max _{h \in N}\left|f_{h}(x)\right| \max _{h \in N}\left|f_{h}(y)\right| .
$$

The most widely used tool for showing the existence of a solution to a system of equations is the Brouwer fixed point theorem or one of its variants. Brouwer's theorem states that any continuous function from a convex and compact subset of $\mathbb{R}^{n}$ to itself leaves at least one point fixed. In models where the domain of interest is a finite set, the continuity and convexity requirement of Brouwer's theorem is no longer fulfilled. In Definition 2.1 the SCOEF condition replaces continuity and convexity is replaced by the notion of an integral convex set. As mentioned in the introduction the issue of existence of a zero point on discrete sets has been addressed recently in several papers under various conditions. Until now in almost all papers the set of interest has been taken to be a cube, in this paper we generalize to an integrally convex set. Further it should be noticed that the class of SCOEF functions includes the class of positive maximum component sign preserving functions considered in Talman and Yang (2009), which class includes the class of direction preserving functions. Also the class of locally gross direction preserving functions includes 
the class direction preserving functions. The following example shows that SCOEF functions and locally gross direction preserving functions are not comparable in the sense that they do not imply each other.

Example 2.2 Let $f: \mathbb{Z}^{6} \rightarrow \mathbb{R}^{6}$ be defined by $f(x)=(2,1,1,1,1,1)$ for $x=(0,0,0,0,0,0)$, $f(x)=(-2,1,1,1,1,1)$ for $x=(1,1,1,1,1,1)$, and $f(x)=(0,0,0,0,0,0)$ otherwise. Then $f$ is locally gross direction preserving but not SCOEF. Let $f: \mathbb{Z}^{2} \rightarrow \mathbb{R}^{2}$ be defined by $f(x)=(2,1)$ for $x=(0,0), f(x)=(-2,-3)$ for $x=(1,1)$, and $f(x)=(0,0)$ otherwise. Then $f$ is SCOEF but not locally gross direction preserving.

We also want to stress that the existence of a locally gross direction preserving function $f$ on a discrete set $D$ can be proved by extending $f$ to a function $\bar{f}$ on the convex hull of $D$ with $\bar{f}$ the piecewise linear approximation of $f$ on a cubical triangulation $\mathcal{T}$ of $D$. Under some boundary condition the existence of a zero point of $\bar{f}$ follows from a standard zero (or fixed) point argument and then the locally gross direction preserving condition guarantees that when $x$ is a zero point of $\bar{f}$ in some simplex $\sigma$ of $\mathcal{T}$, then $\sigma$ contains at least one vertex that is a zero point of $f$ itself. This does not hold on the class of SCOEF functions. The piecewise linear approximation of a SCOEF function may have a zero point, whereas the function itself does not have a zero point. This means that the existence of a discrete zero point of a SCOEF function can not be proven by applying a simplicial vector labeling algorithm, which terminates with a zero point of $\bar{f}$, and neither a standard fixed point argument can be used.

Example 2.3 Let $f: \mathbb{Z}^{3} \rightarrow \mathbb{R}^{3}$ be defined by $f(x)=(2,-1,-1)$ for $x=(1,0,0), f(y)=$ $(-1,2,-1)$ for $y=(0,1,0), f(z)=(-1,-1,2)$ for $z=(0,0,1)$, otherwise $f(w)=(1,1,1)$. Clearly, $f$ is SCOEF. However, $f$ has no discrete zero point, while the barycenter of the triangle with vertices $x, y$ and $z$ is a zero point of the piecewise linear extension of $f$.

\section{Existence of discrete zero point and application}

\subsection{The main result}

In this subsection we present the main theorem on the existence of a discrete zero point of a SCOEF function defined on a finite and regular integrally convex set. Except convexity of the domain set and continuity of the function, standard fixed (or zero) point arguments require compactness and some boundary condition. Within the discrete setting the compactness follows from the finiteness of the domain $D$, the boundary condition in the next theorem can be seen as a discrete version of the standard condition that the function points inwards on the boundary. For a set $X$ in $\mathbb{R}^{n}$, a vector $w \in \mathbb{R}^{n}$ is outgoing at $x \in X$ if 
$x+\epsilon w \notin \bar{X}$ for any $\epsilon>0$. Notice that for a finite and regular integrally convex set $D$, a point $x \in D$ does not have an outgoing vector when $x$ is in the interior of $\bar{D}$.

Theorem 3.1 Let $D$ be a finite and regular integrally convex set in $\mathbb{Z}^{n}$ and let $f: D \rightarrow$ $\mathbb{R}^{n}$ be a SCOEF function satisfying for any $x \in D \cap \partial \bar{D}$ with $f(x) \neq 0^{n}$ that $f_{h}(x)<$ $\max _{i \in N}\left|f_{i}(x)\right|$ if $e(h)$ is outgoing at $x$ for some $h \in N$, and $f_{h}(x)>-\max _{i \in N}\left|f_{i}(x)\right|$ if $e(-h)$ is outgoing at $x$ for some $h \in N$. Then $f$ has a discrete zero point in $D$.

The boundary condition states that if at an integral point $x$ on the boundary of the convex hull of $D$ the $h$ th component of $x$ cannot be increased, i.e., $e(h)$ is an outgoing unit vector at $x$, then the $h$ th component of the function value $f(x)$ does not have positive extreme value, and if the $h$ th component of $x$ cannot be decreased, i.e., $e(-h)$ is an outgoing unit vector at $x$, then the $h$ th component of the function value $f(x)$ does not have negative extreme value.

To prove Theorem 3.1, we implement an integer labeling rule $\ell(\cdot)$ that assigns to each point $x$ of the discrete set $D$ an integer label from the set $N \cup N^{-} \cup\{0\}$, where $N^{-}=$ $\{-1,-2, \ldots,-n\}$.

Definition 3.2 For $f: D \rightarrow \mathbb{R}^{n}$, the labeling function $\ell: D \rightarrow N \cup N^{-} \cup\{0\}$ is as follows. For $x \in D$, take any $k \in N$ such that $\left|f_{k}(x)\right|=\max _{h \in N}\left|f_{h}(x)\right|$. Then, $\ell(x)=0$ if $f_{k}(x)=0, \ell(x)=k$ if $f_{k}(x)>0$, and $\ell(x)=-k$ if $f_{k}(x)<0$.

Notice that $\ell(x)=0$ if and only if $x$ is a discrete zero point of $f$. The following lemma shows that this integer labeling rule excludes the possibility that any two vertices of a simplex are oppositely labeled when the function satisfies the SCOEF property.

Lemma 3.3 Let $f: D \rightarrow \mathbb{R}^{n}$ satisfy the SCOEF conditions of Definition 2.1 for some cubical triangulation $\mathcal{T}$ of $\bar{D}$ and let $\ell(\cdot)$ be the integer labeling rule as given in Definition 3.2. Then there are no two vertices $x$ and $y$ of a same simplex of $\mathcal{T}$ such that $\ell(x)=k$ and $\ell(y)=-k$ for any $k \in N$.

Proof. Suppose to the contrary that there is a simplex in $\mathcal{T}$ with two vertices $x$ and $y$ such that $\ell(x)=k$ and $\ell(y)=-k$ for some $k \in N$. Then, by Definition 3.2, $f(x) \neq 0^{n}$ and $f(y) \neq 0^{n}, f_{k}(x)=\max _{h}\left|f_{h}(x)\right|$ and $f_{k}(y)=-\max _{h}\left|f_{h}(x)\right|$. This contradicts that $f$ satisfies the SCOEF property of Definition 2.1.

The next lemma states that under the boundary condition a vertex cannot carry label $h$ if $e(h)$ is an outgoing vector.

Lemma 3.4 Let $f: D \rightarrow \mathbb{R}^{n}$ satisfy the boundary condition of Theorem 3.1 and let $\ell(\cdot)$ be the integer labeling rule given in Definition 3.2. Then, for any $x \in D \cap \partial \bar{D}, \ell(x) \neq h$ whenever $e(h), h \in N \cup N^{-}$, is an outgoing vector at $x$. 
Proof. Take any $x$ in $D \cap \partial \bar{D}$. Let $k \in N$ be such that $e(k)$ is an outgoing vector at $x$. From the boundary condition it follows that either $f(x)=0^{n}$ or $f_{k}(x)<\max _{h}\left|f_{h}(x)\right|$. In both cases $\ell(x) \neq k$. Next, let $k \in N^{-}$be such that $e(k)$ is an outgoing vector at $x$. From the boundary condition it then follows that either $f(x)=0^{n}$ or $f_{k}(x)>-\max _{h}\left|f_{h}(x)\right|$. Again, in both cases $\ell(x) \neq k$. Therefore, $\ell(x) \neq h$ whenever $e(h), h \in N \cup N^{-}$, is an outgoing vector at $x$.

To give a constructive proof of Theorem 3.1, let

$$
\mathcal{I}=\left\{T \subset N \cup N^{-} \mid k \in T \text { implies }-k \notin T\right\}
$$

be the collection of all subsets of $N \cup N^{-}$not containing two opposite labels. Further, let $v$ be an arbitrarily chosen integral point in the interior of $\bar{D}$. Since $D$ is regular and full-dimensional, such a point $v$ exists. We then define for any $T \in \mathcal{I}$ the set $A(T)$ by

$$
A(T)=\left\{x \in \bar{D} \mid \quad x_{i} \geq v_{i} \text { if } i \in T ; x_{i} \leq v_{i} \text { if }-i \in T \text {; and } x_{i}=v_{i} \text { otherwise }\right\} .
$$

Clearly, $A(\emptyset)=\{v\}$, and for any $T \in \mathcal{I}$ the set $A(T)$ is a $t$-dimensional convex set, where $t=|T|$, satisfying that the $i$ th component of an element $x$ in $A(T)$ is at least (most) as large as the $i$ th component of $v$ when $i \in T(-i \in T)$, and $x_{i}=v_{i}$ when neither $i$ nor $-i$ is in $T$. Let $\mathcal{T}$ be a cubical triangulation of $\bar{D}$ with respect to which $f$ is SCOEF. Since $\mathcal{T}$ is cubical, $\mathcal{T}$ induces for every $T \in \mathcal{I}$ a subdivision of $A(T)$ into $t$-dimensional cubical simplices, with $t=|T|$. Any facet $\tau$ of a $t$-simplex in $A(T)$ is either a facet of exactly one other $t$-simplex in $A(T)$ or lies on the boundary of $A(T)$. In the latter case $\tau$ is either a $(t-1)$-simplex in $A(T \backslash\{k\})$ for some unique $k \in T$ or a facet in $A(T)$ on the boundary of $\bar{D}$. Furthermore, every $t$-simplex in $A(T)$ is cubical and therefore any two vertices of a $t$-simplex in $A(T)$ are cell-connected. Given a $t$-dimensional simplex $\sigma$ in $A(T)$, with $t=|T|$, a facet $\tau$ of $\sigma$ is $T$-complete if

$$
T=\{\ell(x) \mid x \text { is a vertex of } \tau\},
$$

i.e., its $t$ vertices are differently labeled by the $t$ elements of $T$. The next lemma shows that a facet of a simplex in $A(T)$ on the boundary of $\bar{D}$ can not be $T$-complete.

Lemma 3.5 Let $f: D \rightarrow \mathbb{R}^{n}$ satisfy the SCOEF conditions of Definition 2.1 for some cubical triangulation $\mathcal{T}$ of $\bar{D}$ and let $\ell(\cdot)$ be the integer labeling rule as given in Definition 3.2. Then there does not exist a T-complete facet of $\mathcal{T}$ in $A(T) \cap \partial \bar{D}$.

Proof. Suppose that $\tau$ is a $T$-complete facet in $A(T)$ for some $T \in \mathcal{I}$ and that $\tau$ lies on the boundary of $\bar{D}$. Take any point $x$ in the relative interior of $\tau$ and let $K=\{h \in$ $N \cup N^{-} \mid e(h)$ is outgoing at $\left.x\right\}$. Since $D$ is integrally convex, $K \neq \emptyset$. Moreover, for any 
$k \in K, e(k)$ is an outgoing vector at every vertex of $\tau$ and therefore, by Lemma 3.4, no vertex of $\tau$ has label $k$. To prove that $\tau$ can not be $T$-complete, we show that $T \cap K \neq \emptyset$.

Suppose to the contrary that $T \cap K=\emptyset$. Then, no $e(h), h \in T$, is outgoing and thus there exists $\bar{\epsilon}>0$ such that for all $\epsilon, 0 \leq \epsilon \leq \bar{\epsilon}$,

$$
x+\epsilon e(h) \in \bar{D}, \text { for any } h \in T \text {. }
$$

Clearly, $A(T) \cap \partial \bar{D}$ is $(t-1)$-dimensional. So, the $(t-1)$-dimensional $T$-complete facet $\tau \in \partial \bar{D}$ can not be in $A(T \backslash\{h\})$ for any $h \in T$, because $A(T \backslash\{h\}) \cap \partial \bar{D}$ is of dimension $t-2$. Hence, the relative interior of $\tau$ is in the relative interior of $A(T)$, and so is $x$, implying that there exist unique $\lambda_{h}>0, h \in T$, such that

$$
x=v+\sum_{h \in T} \lambda_{h} e(h) .
$$

For some $\epsilon, 0<\epsilon \leq \bar{\epsilon}$, let

$$
y=\frac{1}{1-\lambda}(x-\lambda v),
$$

where $\lambda=\frac{\epsilon}{\epsilon+\mu}$ with $\mu=\sum_{h \in T} \lambda_{h}$. Then substituting $v=x-\sum_{h \in T} \lambda_{h} e(h)$ in equation (3.1) yields

$$
\begin{gathered}
y=\frac{1}{1-\lambda}\left(x-\lambda\left(x-\sum_{h \in T} \lambda_{h} e(h)\right)=x+\frac{\lambda}{1-\lambda} \sum_{h \in T} \lambda_{h} e(h)\right) \\
=x+\frac{\epsilon}{\mu} \sum_{h \in T} \lambda_{h} e(h)=\sum_{h \in T} \frac{\lambda_{h}}{\mu}(x+\epsilon e(h)) .
\end{gathered}
$$

Since $x+\epsilon e(h) \in \bar{D}$ for every $h \in T$ and $\mu=\sum_{h \in T} \lambda_{h}$, it follows that $y$ is a convex combination of elements of $\bar{D}$ and thus $y \in \bar{D}$, because $\bar{D}$ is convex. On the other hand, equation (3.1) yields $x=\lambda v+(1-\lambda) y$ with $0<\lambda=\frac{\epsilon}{\epsilon+\mu}<1$. Since $v$ is in the interior of $\bar{D}$ and $y$ is in $\bar{D}$, it follows that $x$ is in the interior of $\bar{D}$, contradicting that $x$ lies on the boundary of $\bar{D}$. Consequently, $T \cap K \neq \emptyset$ and therefore $\tau$ is not $T$-complete.

We now apply the $2 n$-ray variable dimension algorithm of van der Laan and Talman (1981) and Reiser (1981) in $\mathbb{R}^{n}$ on the set $\bar{D}$ to find a zero point of $f$. In the original algorithm the sets $A(T)$ are defined as subsets in $\mathbb{R}^{n}$ and some convergency condition guarantees that the algorithm stays within a bounded subset of $\mathbb{R}^{n}$. Here the sets $A(T)$ are defined as subsets of $\bar{D}$ and the boundary condition replaces the convergency condition.

\section{The 2n-ray algorithm.}

Step 0. Set $T=\emptyset, x=v, \sigma=\{v\}$, and go to Step 1 . 
Step 1. Calculate $\ell(x)$. If $\ell(x)=0$, stop. If $\ell(x) \in T$, go to Step 2. Otherwise, go to Step 3.

Step 2. Let $\tau$ be the facet of $\sigma$ opposite to vertex $x$. If $\tau$ is in $A(T \backslash\{k\})$ for some $k \in T$, go to Step 4. Otherwise, let $\sigma^{\prime}$ be the unique simplex in $A(T)$ sharing $\tau$ with $\sigma$, then $\sigma$ becomes $\sigma^{\prime}, x$ becomes the vertex of $\sigma^{\prime}$ opposite to $\tau$, and go to Step 1 .

Step 3. Set $h=\ell(x)$ and let $\sigma^{\prime}$ be the unique simplex in $\left.A(T \cup\{h\})\right)$ having $\tau$ as facet, then $T$ becomes $T \cup\{h\}, \sigma$ becomes $\sigma^{\prime}, x$ becomes the vertex of $\sigma^{\prime}$ opposite to $\tau$, and go to Step 1.

Step 4. $T$ becomes $T \backslash\{k\}, \sigma$ becomes $\tau, x$ becomes the vertex of $\tau$ with label $k$, and go to Step 2.

We now show that the algorithm indeed terminates with a zero point of $f$ within a finite number of steps, which proves Theorem 3.1.

Proof of Theorem 3.1. The algorithm starts at $v$ in $A(T)$ with $T=\emptyset$. If $\ell(v)=0$, then $v$ is a discrete zero point of $f$ and the algorithm immediately terminates in Step 1. If $\ell(v) \neq 0$, then, since $\ell(v) \notin T$, the algorithm goes to Step 3 and $T$ becomes $\{\ell(v)\}$. Then $\{v\}$ is a 0 -dimensional facet of a unique 1-dimensional simplex $\sigma^{0}$ in $A(T)$, where $\sigma^{0}=\sigma\left(x^{1}, x^{2}\right)$ with $x^{1}=v$ and $x^{2}=v+e(\ell(v))$, and the algorithm goes back to Step 1 with $x$ equal to the vertex $x^{2}$. The algorithm stops when $\ell\left(x^{2}\right)=0$. Otherwise, when $\ell\left(x^{2}\right) \neq \ell(v)$, then the algorithm goes again to Step 3 and $T$ becomes $\left\{\ell(v), \ell\left(x^{2}\right)\right\}$. When $\ell\left(x^{2}\right)=\ell(v)$, the algorithm goes to Step 2 and starting with $\sigma^{0}$ the algorithm generates a sequence of adjacent 1-dimensional simplices in $A(T)$ with $T$-complete common facets where $T=\{\ell(v)\}$. More general, for varying sets $T \in \mathcal{I}$, the algorithm generates by performing alternating Steps 1 and 2 a sequence of adjacent $t$-simplices in $A(T)$ with $T$ complete common facets, where $t=|T|$. When a simplex is generated having a vertex with label 0, the algorithm stops in Step 1. Otherwise, the set $T$ is adapted when either (i) in Step 1 a $t$-simplex $\sigma$ in $A(T)$ is encountered having a vertex with a label $h \neq 0$ that does not belong to $T$, or (ii) in Step 2 a simplex in $A(T)$ is encountered having a $T$-complete facet $\tau$ in $A(T \backslash\{k\})$ for some $k \in T$. In case (i), it follows from Lemma 3.3 that the new found label $h$ can not be the negative of a label that belongs to $T$, and thus $T^{\prime}=T \cup\{h\} \in \mathcal{I}$. Now, the algorithm goes to Step 3 and the algorithm continues in $A\left(T^{\prime}\right)$, starting from the unique $(t+1)$-simplex in $A\left(T^{\prime}\right)$ having $\sigma$ as its facet. In case (ii), the algorithm goes to Step 4, where label $k$ is deleted from $T$ and the algorithm continues in $A(T \backslash\{k\})$, starting with the $(t-1)$-simplex that is the facet opposite the vertex of $\tau$ carrying label $k$. Since each step of the algorithm is uniquely determined, it follows by the well-known Lemke-Howson argument that a simplex will never be visited more than once 
(see van der Laan and Talman (1981) or Reiser (1981) for a more detailed description). Since the number of simplices in the triangulation of $\bar{D}$ is finite, within a finite number of steps the algorithm either terminates in Step 1 with a vertex having label 0, or generates, for some $T \in \mathcal{I}$, a simplex in $A(T)$ having a $T$-complete facet $\tau$ in $A(T) \cap \partial \bar{D}$. By Lemma 3.5 , the latter case can not occur and thus the algorithm terminates within a finite number of steps with a simplex having a vertex $x$ carrying label 0 . Since all vertices of any simplex generated by the algorithm are elements of $D, x$ is a discrete zero point of $f$.

Theorem 3.1 holds for any finite (regular) integrally convex set $D$. A special case is that $\bar{D}$ is an $n$-dimensional rectangular set.

Corollary 3.6 Let $f: \mathbb{Z}^{n} \rightarrow \mathbb{R}^{n}$ be a SCOEF function, satisfying that there exist $a, b \in \mathbb{Z}^{n}$ with $b_{i}>a_{i}+1$ for every $i \in N$, such that for $x \in \mathbb{Z}^{n}$ with $f(x) \neq 0^{n}$ it holds that (i) if $x_{j}=a_{j}$, then $f_{j}(x)>-\max _{h \in N}\left|f_{h}(x)\right|$, and (ii) if $x_{j}=b_{j}$, then $f_{j}(x)<\max _{h \in N}\left|f_{h}(x)\right|$. Then $f$ has a discrete zero point in the finite set $D=\left\{x \in \mathbb{Z}^{n} \mid a_{i} \leq x_{i} \leq b_{i}, i \in N\right\}$.

In particular observe that the boundary conditions of Corollary 3.6 are satisfied when the function $f$ is pointing inwards on the boundary of the rectangular set $\bar{D}$, i.e., $f_{j}(x) \geq 0$ if $x_{j}=a_{j}$ and $f_{j}(x) \leq 0$ if $x_{j}=b_{j}$.

\subsection{Application: the discrete oligopoly model}

Now we turn to the application of a Cournot oligopoly. Suppose there are $n$ producers of a homogeneous commodity with inverse market demand function specified by

$$
P(q)=a-b q, \quad q \in \mathbb{Z}_{+},
$$

where $a$ and $b$ are strictly positive real numbers. Firm $i, i \in N$, has a quadratic cost function $c_{i}: \mathbb{Z}_{+} \rightarrow \mathbb{R}$ given by

$$
c_{i}\left(q_{i}\right)=c_{i} q_{i}+\frac{d_{i}}{2}\left(q_{i}\right)^{2}, \quad q_{i} \in \mathbb{Z}_{+},
$$

where $c_{i}$ and $d_{i}$ are real numbers. We typically think of $c_{i}$ and $d_{i}$ as being non-negative, but this is not required. What we do require is $2 b+d_{i}>0$, since otherwise it would be optimal for firm $i$ to produce infinite amounts. Firm $i \in N$ maximizes his profit given the quantities set by its competitors and thus faces the maximization problem

$$
\max _{q_{i} \in \mathbb{\mathbb { Z }}_{+}}\left(a-b q_{i}-b \sum_{j \neq i} q_{j}\right) q_{i}-\left(c_{i} q_{i}+\frac{d_{i}}{2}\left(q_{i}\right)^{2}\right) .
$$

Treating $q_{i}$ as a real variable in $\mathbb{R}$, the first order condition is

$$
a-2 b q_{i}-b \sum_{j \neq i} q_{j}-c_{i}-d_{i} q_{i}=0
$$


so firm $i$ 's optimal production quantity $\bar{q}_{i}$ in $\mathbb{R}$, given the quantities $q_{j}, j \neq i$, of its competitors, is given by

$$
\bar{q}_{i}=\frac{a-c_{i}}{2 b+d_{i}}-\frac{b}{2 b+d_{i}} \sum_{j \neq i} q_{j} .
$$

For a real number $x$, let $[x]$ denote the integer that results from the usual way of rounding off, i.e., $[x]$ is the integer nearest to $x$ if the nearest integer is uniquely determined, and the highest such integer otherwise.

Lemma 3.7 The quantity $q_{i}^{*}$ given by

$$
q_{i}^{*}=\max \left\{0,\left[\bar{q}_{i}\right]\right\}
$$

is firm ith optimal integer production, given the (integer) production quantities $q_{j}, j \neq i$, of its competitors.

Proof. Let $g: \mathbb{R} \rightarrow \mathbb{R}$ be a quadratic function given by

$$
g(x)=\alpha x^{2}+\beta x+\gamma,
$$

with $\alpha<0$. Its maximum on $\mathbb{R}$ is obtained for $\bar{x}=-\beta /(2 \alpha)$ and it follows straightforwardly that

$$
g(x)=\alpha\left(x-x^{*}\right)^{2}+g(\bar{x}) .
$$

It is now seen easily that the non-negative integer nearest to $\bar{x}$, i.e., $x^{*}=\max \{0,[\bar{x}]\}$ is a solution to the maximization problem of $g$ on $\mathbb{Z}_{+}$. Since the objective function of firm $i$, $i \in N$, is a function of type $g$, it follows that $q_{i}^{*}=\max \left\{0,\left[\bar{q}_{i}\right]\right\}$ maximizes firm $i$ 's objective function over $\mathrm{Z}_{+}$.

Notice that in case $\bar{q}_{i}$ equals a nonnegative integer plus $1 / 2$, also $q_{i}^{*}-1$ maximizes firm $i$ 's objective function on $\mathbb{Z}_{+}$. However, Lemma 3.7 allows us to define for $i \in N$ the function $r^{i}: \mathbb{Z}_{+}^{n} \rightarrow \mathbb{Z}_{+}$given by $r^{i}(q)=\max \left\{0,\left[\bar{q}_{i}\right]\right\}$ as a selection of firm $i$ 's optimal reaction to the quantities $q_{j}, j \neq i$, of its competitors. A closer inspection of the function $r^{i}$ reveals that $\left[\frac{a-c_{i}}{2 b+d_{i}}\right]$ is the maximal amount firm $i$ will ever choose as its optimal reaction. So, when searching for a Cournot-Nash equilibrium in which every player $i$ chooses a quantity that is optimal against the quantities set by its competitors, we may restrict attention to the set of discrete strategy combinations $D=\left\{x \in \mathbb{Z}^{n} \mid l \leq x \leq u\right\}$ with $l_{i}=0$ and $u_{i}=\max \left\{2, \frac{a-c_{i}}{2 b+d_{i}}\right\}, i \in N$.

Let $f: D \rightarrow \mathbb{R}^{n}$ be given by

$$
f_{j}(q)=r^{j}(q)-q_{j}, \quad j \in N, \quad q \in D .
$$


It now follows that, when $b+d_{i}>0$ for all $i$, the discrete oligopoly model has a CournotNash equilibrium in $D$ by showing that $f$ is simplicially coordinatewise opposite extrema free. For this, we take the $H$-triangulation of $\mathbb{R}^{n}$ with grid size equal to 1 (see Saigal (1983)), restricted to $\bar{D}=\left\{x \in \mathbb{R}^{n} \mid l \leq x \leq u\right\}$. This triangulation is a cubical triangulation and is defined as follows. Let the vectors $p^{h} \in \mathbb{R}^{n}, h \in N$, be given by $p^{1}=(1,0, \ldots, 0)^{\top}$ and, for $h=2, \ldots, n, p^{h}=(0, \ldots, 0,-1,1,0, \ldots, 0)^{\top}$ with 1 on the $h$ th place.

Definition 3.8 For $y \in \mathbb{Z}^{n}$ and a permutation $\pi=(\pi(1), \ldots, \pi(n))$ of the elements in $\{1, \ldots, n\}$, let $\sigma(y, \pi)$ be the convex hull of the vectors $y^{0}, y^{1}, \ldots, y^{n}$ given by

$$
y^{0}=y \text { and } y^{h}=y^{h-1}+p^{\pi(h)}, \quad h \in N .
$$

Then the cubical H-triangulation of $\mathbb{R}^{n}$ restricted to $\bar{D}$ is the collection of all simplices $\sigma(y, \pi)$ that belong to $\bar{D}$.

We call the cubical $H$-triangulation of $\mathbb{R}^{n}$ restricted to $\bar{D}$ for simplicity the cubical $H$ triangulation of $\bar{D}$.

Theorem 3.9 If $b+d_{i} \geq 0, i \in N$, then $f: D \rightarrow \mathbb{R}^{n}$ satisfies the SCOEF conditions of Definition 2.1 for the cubical $H$-triangulation of $\bar{D}$.

Proof. For $i \in N$ and $q \in D$ it holds that

$$
\begin{aligned}
f_{i}(q) & =r^{i}(q)-q_{i}=\max \left\{0,\left[\frac{a-c_{i}}{2 b+d_{i}}-\frac{b}{2 b+d_{i}} \sum_{j \neq i} q_{j}\right]\right\}-q_{i} \\
& =\max \left\{-q_{i},\left[\frac{a-c_{i}}{2 b+d_{i}}-\frac{b}{2 b+d_{i}} \sum_{j \neq i} q_{j}-q_{i}\right]\right\} \\
& =\max \left\{-q_{i},\left[\frac{a-c_{i}}{2 b+d_{i}}-\left(\frac{b}{2 b+d_{i}} \sum_{j=1}^{n} q_{j}+\frac{b+d_{i}}{2 b+d_{i}} q_{i}\right)\right]\right\} .
\end{aligned}
$$

Consider any two vertices $q^{h}$ and $q^{k}$ of a simplex $\sigma$ and take any $i \in N$. By the $H$ triangulation it holds that

$$
\left|q_{i}^{h}-q_{i}^{k}\right| \leq 1 \text { and }\left|\sum_{j=1}^{n} q_{j}^{h}-\sum_{j=1}^{n} q_{j}^{k}\right| \leq 1 .
$$

Since $b+d_{i}>0$ and thus $0<b /\left(2 b+d_{i}\right) \leq 1$, it follows that

$$
\left|\left(\frac{b}{2 b+d_{i}} \sum_{j=1}^{n} q_{j}^{h}+\frac{b+d_{i}}{2 b+d_{i}} q_{i}^{h}\right)-\left(\frac{b}{2 b+d_{i}} \sum_{j=1}^{n} q_{j}^{k}+\frac{b+d_{i}}{2 b+d_{i}} q_{i}^{k}\right)\right| \leq 1
$$


and consequently that

$$
\left|f_{i}\left(q^{h}\right)-f_{i}\left(q^{k}\right)\right| \leq 1
$$

Hence $f_{i}\left(q^{h}\right)$ and $f_{i}\left(q^{k}\right)$ are both non-negative or both non-positive, and so $f: D \rightarrow \mathbb{R}^{n}$ satisfies the SCOEF condition of Definition 2.1.

Theorem 3.10 If $b+d_{i} \geq 0, i \in N$, then the discrete oligopoly model has a discrete Cournot-Nash equilibrium.

Proof. By Theorem 3.9 the function $f$ is SCOEF on the set $D$. Moreover, $f_{i}(x)>0$ if $x_{i}=0$ and $f_{i}(x)<0$ if $x_{i}=\max \left\{2, \frac{a-c_{i}}{2 b+d_{i}}\right\}$. So, by Corollary 3.6, $f$ has a discrete zero point $q^{*}$ in the set $D$. Since $f\left(q^{*}\right)=0^{n}$ implies that $q_{i}^{*}=r^{i}\left(q^{*}\right), i \in N$, it follows that $q^{*}$ is a Cournot-Nash equilibrium in $D$.

It should be noticed that a function has the SCOEF property if there exists a cubical triangulation for which the conditions of Definition 2.1 hold. In the oligopoly model the use of the $H$-triangulation is crucial, the next example shows that the function $f$ may not satisfy the conditions of Definition 2.1 for other cubical triangulations.

Example 3.11 Consider a discrete Cournot oligopoly model with two firms having zero production costs and inverse demand function $P(q)=9-2 q, q \in \mathbb{Z}_{+}$. The best response of firm $i, i=1,2$, is given by $r^{i}(q)=2$ when $q_{j} \in\{0,1\}, r^{i}(q)=1$ when $q_{j} \in\{2,3\}$ and $r^{i}(q)=0$ for any other $q_{j} \in \mathbb{Z}_{+}$, where $q_{j}$ is $i$ 's competitor's quantity. Clearly, the function $f$ given by $f(q)=\left(r^{1}(q)-q_{1}, r^{2}(q)-q_{2}\right)^{\top}$ is SCOEF for the $H$-triangulation of $\bar{D}$ and there are two discrete Cournot-Nash equilibria, namely $q^{*}=(2,1)^{\top}$ and $q^{* *}=(1,2)^{\top}$. The cubical $K$-triangulation of $\mathbb{R}^{n}$ is the collection of simplices that are the convex hull of vectors $y^{0}, y^{1}, \ldots, y^{n}$ that, for some $y \in \mathbb{Z}^{n}$ and permutation $\pi=(\pi(1), \ldots, \pi(n))$ of the elements in $N$, are given by

$$
y^{0}=y \text { and } y^{h}=y^{h-1}+e(\pi(h)), \quad h=1, \ldots, n .
$$

For $y=(1,1)^{\top}$ and both permutations on $N=\{1,2\}$, it follows that $y^{0}=(1,1)^{\top}$ and $y^{2}=$ $(2,2)^{\top}$. Therefore, both elements $y^{0}=(1,1)^{\top}$ and $y^{2}=(2,2)^{\top}$ of $D$ are in a same simplex of the cubical $K$-triangulation. Since $f\left(y^{0}\right)=(1,1)^{\top}$ and $f\left(y^{2}\right)=(-1,-1)^{\top}$, it follows that $f$ does not satisfy the conditions of Definition 2.1 for the cubical $K$-triangulation restricted to $\bar{D}$. Notice that indeed $y^{0}$ and $y^{2}$ are not vertices of one simplex of the cubical $H$-triangulation of $\bar{D}$. 


\section{More existence results for SCOEF functions}

\subsection{A discrete version of the Borsuk-Ulam theorem}

The next theorem might be viewed as a discrete analogue of the famous Borsuk-Ulam theorem for continuous functions. Notice that as usual it is required that the domain set $D$ is symmetric (around the origin), i.e., $x \in D$ implies $-x \in D$

Theorem 4.1 Let $D \subset \mathbb{Z}^{n}$ be a finite, regular and symmetric integrally convex set and let $f: D \rightarrow \mathbb{R}^{n}$ be a function that satisfies the SCOEF conditions of Definition 2.1 for some symmetric cubical triangulation $\mathcal{T}$ of $\bar{D}$. Suppose that for any $x \in \partial \bar{D} \cap D$ there exists some $k \in N$ such that $f_{k}(x) f_{k}(-x)=-\max _{i \in N}\left|f_{i}(x)\right| \max _{i \in N}\left|f_{i}(-x)\right|$. Then $f$ has a discrete zero point in $D$.

The boundary condition in this theorem might seem to be rather strong, but it is actually much weaker than the standard boundary condition for the Borsuk-Ulam theorem, namely that $f(-x)=-f(x)$ when $x$ is on the boundary of the domain set. To prove Theorem 4.1, when $x \in D$ in the interior of $\bar{D}$, the label $\ell(x)$ is as given in Definition 3.2. When $x$ is a point in $\partial \bar{D} \cap D$, the labeling is slightly modified as follows. If $f(x)=0^{n}$, then $\ell(x)=0$. If $f(x) \neq 0^{n}$ and $f(-x)=0^{n}$, then $\ell(x)$ is as given in Definition 3.2. If $f(x) \neq 0^{n}$ and $f(-x) \neq 0^{n}$, then, according to the boundary condition there exists $k \in N$ such that $f_{k}(x) f_{k}(-x)=-\max _{i \in N}\left|f_{i}(x)\right| \max _{i \in N}\left|f_{i}(-x)\right|$. Take any of these, and set $\ell(x)=k$ if $f_{k}(x)>0$ and $\ell(x)=-k$ if $f_{k}(x)<0$. Clearly, the modified labeling rule $\ell(\cdot)$ satisfies that two antipodal points $x$ and $-x$ on the boundary of $\bar{D}$ either have opposite labels or one of the two points has label 0 . The next lemma shows that no two vertices of a same simplex can have opposite labels.

Lemma 4.2 Let $f: D \rightarrow \mathbb{R}^{n}$ satisfy the conditions of Theorem 4.1. Then there is no simplex in $\mathcal{T}$ carrying both labels $k$ and $-k$ for any $k \in N$.

Proof. Let $x$ and $y$ be two vertices of any simplex of $\mathcal{T}$. When both $x$ and $y$ are in the interior of $\bar{D}$, then similarly as in the proof of Lemma 3.4 it follows that $x$ and $y$ can not have opposite labels. So, let $x$ be a vertex on the boundary of $\bar{D}$ with $\ell(x) \neq 0$. Without loss of generality, suppose that $\ell(x)=k$ for some $k \in N$. From the modified labeling rule it follows that $f_{k}(x)>0$ and $f_{k}(x) f_{k}(-x)=-\max _{i \in N}\left|f_{i}(x)\right| \max _{i \in N}\left|f_{i}(-x)\right|$. This implies that $f_{k}(x)=\max _{i \in N}\left|f_{i}(x)\right|$. We now consider two cases. First, when $y$ is in the interior of $\bar{D}$, it follows by the SCOEF condition that $y$ can not have label $-k$. The second case is that also $y$ is on the boundary of $\bar{D}$. Since $D$ is regular and thus $\bar{D}$ has an interior point, two opposite points on the boundary can not belong to the same cell and therefore also not to the same simplex of $\mathcal{T}$. Hence, $y$ is not opposite to $x$, i.e., 
$y \neq-x$. Suppose that $y$ has label $-k$. Then, by the labeling rule we must have that $f_{k}(y)<0$ and $f_{k}(y) f_{k}(-y)=-\max _{i \in N}\left|f_{i}(y)\right| \max _{i \in N}\left|f_{i}(-y)\right|$. However, this implies that $f_{k}(y)=-\max _{i \in N}\left|f_{i}(y)\right|$, which contradicts the SCOEF condition.

To prove Theorem 4.1 we now take the origin as the starting point $v$ of the $2 n$-ray algorithm. So for any $T \in \mathcal{I}$ the set $A(T)$ is defined by

$$
A(T)=\left\{x \in \bar{D} \mid x_{i} \geq 0 \text { if } i \in T ; x_{i} \leq 0 \text { if }-i \in T ; \text { and } x_{i}=0 \text {, otherwise }\right\} .
$$

Since the triangulation $\mathcal{T}$ for which $f$ satisfies the SCOEF conditions is cubical, it induces for every $T \in \mathcal{I}$ a subdivision of $A(T)$ into $t$-dimensional cubical simplices, where $t=|T|$. Moreover, by the symmetry of $D$ it follows that $A(-T)=-A(T)$ and by the symmetry of $\mathcal{T}$ that if $\sigma$ is a $t$-simplex in $A(T)$, then $-\sigma$ is a $t$-simplex in $A(-T)$. In particular, it holds that when $\tau$ is a facet of a $t$-simplex $\sigma$ in $A(T)$ lying on the boundary of $\bar{D}$, then $-\tau$ also lies on the boundary of $\bar{D}$ and $-\sigma$ is the only $t$-simplex in $A(-T)$ having $-\tau$ as facet.

Proof of Theorem 4.1. We apply the $2 n$-algorithm starting from $v=0^{n}$ with a minor modification. A similar modification has been used by Freund and Todd (1981) and van der Laan (1984). The algorithm generates a unique sequence of adjacent $t$-simplices in $A(T)$ with $T$-complete common facets for varying $T \in \mathcal{I}$ according to the Steps 1-4 with the modification that when in Step 2 a $T$-complete facet $\tau$ of a simplex $\sigma$ in $A(T)$ is generated that lies on the boundary of $\bar{D}$, then first the labels of the vertices of the opposite facet $-\tau$ are investigated. If (at least) one of these labels is zero, then a zero point of $f$ has been found and the algorithm stops. Otherwise, the label of any vertex of $-\tau$ is opposite to the label of its corresponding opposite vertex of $\tau$ and a reflection step is made, i.e., the set $T$ becomes $-T$, $\tau$ becomes $-\tau$ and the algorithm goes to Step 3 with the unique simplex in $A(-T)$ having the new $\tau$ as its facet. Notice that this simplex is equal to $-\sigma$.

By Lemma 4.2 there does not exist a simplex having two vertices with opposite label. Similarly as in the proof of Theorem 3.1 it follows that every step is well-defined and each step of the algorithm is uniquely determined. Therefore it follows by the Lemke-Howson argument that no simplex can be visited more than once and the algorithm terminates within a finite number of steps, because the number of simplices is finite. Moreover, when a $T$-complete facet $\tau$ on the boundary of $\bar{D}$ is generated, then either one of the vertices of $-\tau$ has label zero and the algorithm stops, or the algorithm makes a reflection step. Therefore the algorithm always terminates with a simplex having a vertex $x$ with label 0 . Since all vertices of any simplex generated by the algorithm are elements of $D, x$ is a discrete zero point of $f$. 


\subsection{The Discrete Complementarity Problem}

In this section we consider the so-called discrete nonlinear complementarity problem. Given a function $f: \mathbb{R}_{+}^{n} \rightarrow \mathbb{R}^{n}$, the nonlinear complementarity problem (NLCP) is to find a point $x^{*} \in \mathbb{R}_{+}^{n}$ such that

$$
f\left(x^{*}\right) \geq 0^{n} \text { and } x^{*} \cdot f\left(x^{*}\right)=0 .
$$

This problem has long been one of the most important problems in the field of mathematical programming and has been intensively studied for the case where $f$ is a continuous function; see for example Cottle, Pang and Stone (1992), Facchinei and Pang (2003) and Kojima, Megiddo, Noma and Yoshise (1991). The discrete counterpart of this problem is to replace the domain $\mathbb{R}_{+}^{n}$ by the discrete lattice $\mathbb{Z}_{+}^{n}$ and is called the discrete complementarity problem.

In the following we establish a theorem on the existence of solutions to the discrete complementarity problem. For $z \in \mathbb{Z}_{+}^{n}$, let $S^{+}(z)=\left\{h \in N \mid z_{h}>0\right\}$.

Definition 4.3 A function $f: \mathbb{Z}_{+}^{n} \rightarrow \mathbb{R}^{n}$ is simplicially coordinatewise opposite extrema free (SCOEF) on $\mathbb{Z}_{+}^{n}$ if there exists a cubical triangulation $\mathcal{T}$ of $\mathbb{R}_{+}^{n}$ such that for any two vertices $x$ and $y$ of a same simplex of $\mathcal{T}$ there does not exist $k \in S^{+}(x)$ such that

$$
f_{k}(x) f_{k}(y)=-\alpha(x) \alpha(y)<0,
$$

where, for $z \in \mathbb{Z}_{+}^{n}, \alpha(z)=\max \left\{\max _{h \in S^{+}(z)}\left|f_{h}(z)\right|, \max _{h \notin S^{+}(z)}-f_{h}(z)\right\}$.

To solve the discrete complementarity problem, the following labeling rule is implemented.

Definition 4.4 For a function $f: \mathbb{Z}_{+}^{n} \rightarrow \mathbb{R}^{n}$, the labeling function $\ell: \mathbb{Z}_{+}^{n} \rightarrow N \cup N^{-} \cup\{0\}$ is given as follows. Take any $x \in \mathbb{Z}_{+}^{n}$. If $\alpha(x) \leq 0$, then $\ell(x)=0$. If $\alpha(x)>0$, take any $k \in N$ such that $f_{k}(x)=-\alpha(x)$ or both $f_{k}(x)=\alpha(x)$ and $k \in S^{+}(x)$. Then $\ell(x)=k$ if $f_{k}(x)>0$ and $\ell(x)=-k$ if $f_{k}(x)<0$.

The next lemma shows that a point with label 0 solves the discrete complementarity problem with respect to $f$.

Lemma 4.5 If $\ell(x)=0$ for some $x \in \mathbb{Z}_{+}^{n}$, then $x$ is a solution to the discrete complementarity problem.

Proof. Let $x \in \mathbb{Z}_{+}^{n}$ be such that $\ell(x)=0$. Then $\alpha(x) \leq 0$ and therefore $f_{h}(x)=0$ if $x_{h}>0$ and $f_{h}(x) \geq 0$ if $x_{h}=0$. 
Lemma 4.6 Let $f: \mathbb{Z}^{n} \rightarrow \mathbb{R}^{n}$ satisfy the SCOEF conditions of Definition 4.3 for some cubical triangulation $\mathcal{T}$ of $\mathbb{R}^{n}$ and let $\ell(\cdot)$ be the integer labeling rule as given in Definition 4.4. Then there is no simplex in $\mathcal{T}$ whose vertices carry both labels $k$ and $-k$ for some $k \in N$.

Proof. Suppose that simplex $\sigma$ in $\mathcal{T}$ has vertices carrying labels $k$ and $-k$ for some $k \in N$. Let $x$ be a vertex of $\sigma$ carrying label $k$ and let $y$ be a vertex of $\sigma$ carrying label $-k$. From $\ell(x)=k$ it follows that $k \in S^{+}(x)$ and $f_{k}(x)=\alpha(x)>0$. From the SCOEF condition it then follows that $f_{k}(y)>-\alpha(y)$ when $k \in S^{+}(y)$ and $\alpha(y)>0$, and therefore $\ell(y) \neq-k$.

Theorem 4.7 Let $f: \mathbb{Z}_{+}^{n} \rightarrow \mathbb{R}^{n}$ be SCOEF on $\mathbb{Z}_{+}^{n}$. If there exists a vector $u \in \mathbb{Z}^{n}$ with $u_{h}>1$ for every $h$ such that for any $x \in \mathbb{Z}_{+}^{n}$ with $x \leq u, x_{k}=u_{k}$ implies $f_{k}(x) \geq$ $\min \left\{0, \min _{h \in N} f_{h}(x)\right\}$, then the discrete complementarity problem has a solution.

To prove Theorem 4.7, we take any point $v \in \mathbb{Z}_{+}^{n}$ satisfying $0<v_{i}<u_{i}$ for all $i \in N$. The point $v$ is the starting point for the $2 n$-ray algorithm applied to the set $\mathbb{Z}_{+}^{n}$. For $T \in \mathcal{I}$, the set $A(T)$ is defined by

$$
A(T)=\left\{x \in \mathbb{R}_{+}^{n} \mid x_{i} \leq v_{i} \text { if } i \in T ; x_{i} \geq v_{i} \text { if }-i \in T ; \text { and } x_{i}=v_{i} \text { otherwise }\right\} .
$$

Notice that the sets are now defined in the opposite direction compared with the sets we used until now. For any $T \in \mathcal{I}$ it holds that the $i$ th component of an element $x$ in $A(T)$ is not larger (smaller) than the $i$ th component of $v$ when $i \in T(-i \in T)$. The reason is that, contrary to before, we should decrease (increase) the value of a variable $x_{j}$ when $f_{j}(x)$ is positive (negative) in order to get a solution.

Proof of Theorem 4.7. Starting at $v$ the $2 n$-algorithm described above generates, for varying sets $T \in \mathcal{I}$, a unique sequence of adjacent $t$-simplices in $A(T)$ with $T$-complete common facets, where $t=|T|$. When the algorithm generates a simplex having a vertex with a label $h$ not in $T$, then it follows from Lemma 4.6 that the opposite label $-h$ does not belong to $T$, and thus all steps are feasible. We now show that the algorithm stays within the bounded set $U=\left\{x \in \mathbb{R}^{n} \mid 0 \leq x_{i} \leq u_{i}, i \in N\right\}$.

First, suppose the algorithm generates a $T$-complete facet $\tau$ of a $t$-simplex in $A(T)$ in the boundary of $U$ at which $x_{k}=u_{k}$ for some $k \in N$. Since $x_{k}=u_{k}$ for all $x \in \tau$ it holds that label $-k$ is an element of $T$. Because $\tau$ is $T$-complete, there exists a vertex $y$ of $\tau$ carrying label $-k$. Since $y_{k}=u_{k}$, it follows from the boundary condition that $f_{k}(y) \geq \min \left\{0, \min _{h \in N} f_{h}(y)\right\}$, so that $y$ cannot carry label $-k$, which is a contradiction. Second, suppose the algorithm generates a $T$-complete facet $\tau$ of a $t$-simplex in $A(T)$ such 
that $\tau$ lies in the boundary of $U$ at which $x_{k}=0$ for some $k \in N$. Since $x_{k}=0$ for all $x \in \tau$ it holds that label $k$ is an element of $T$. Because $\tau$ is $T$-complete, there exists a vertex $y$ of $\tau$ carrying label $k$. Since $y_{k}=0$, it follows from the labeling function that $y$ cannot carry label $k$, which is a contradiction.

It follows that the algorithm cannot cross the boundary of the set $U$. Since the number of simplices of $\mathcal{T}$ in $U$ is finite, the algorithm terminates within a finite number of steps with a simplex having a vertex $x$ with label 0 . Since the triangulation is cubical and thus every vertex belongs to $Z_{+}^{n}$, it follows by Lemma 4.5 that $x$ is a solution of the discrete complementarity problem.

\section{References}

[1] Cottle, R.W., Pang, J.S., Stone, R.E., 1992. The Linear Complementarity Problem, Academic Press, New York.

[2] Danilov, V., Koshevoy, G., 2004. Existence theorem of zero point in a discrete case, preprint.

[3] Eaves, B.C., 1972. Homotopies for computation of fixed points, Mathematical Programming 3, 1-22.

[4] Facchinei, F., Pang, J.-S., 2003. Finite-Dimensional Variational Inequalities and Complementarity Problems, Vol. I, Vol. II, Springer, New York.

[5] Favati, P., Tardella, F., 1990. Convexity in nonlinear integer programming, Ricerca Operativa 53, 3-44.

[6] Freund, R.M., Todd, M.J., 1981. A constructive proof of Tucker's combinatorial lemma, Journal of Combinatorial Theory 30, 321-325.

[7] Fujishige, S., 2005. Submodular Functions and Optimization, 2nd edition, Elsevier, Amsterdam.

[8] Iimura, T., 2003. A discrete fixed point theorem and its applications, Journal of Mathematical Economics 39, 725-742.

[9] Iimura, T., Murota, K., Tamura, A., 2005. Discrete fixed point theorem reconsidered, Journal of Mathematical Economics 41, 1030-1036.

[10] Iimura, T., Yang, Z. 2005. A study on the demand and response correspondences in the presence of indivisibilities, preprint, Yokohama National University, Yokohama. 
[11] Kojima, M., Megiddo, N., Noma, T., Yoshise, A., 1991. A Unified Approach to Interior Point Algorithms for Linear Complementarity Problems, Springer-Verlag, Berlin.

[12] Kuhn, H.W., 1969. Simplicial approximation of fixed points, Proc. Nat. Acad. of Sci. 61 1238-1242.

[13] Laan, G. van der, 1984. On the existence and approximation of zeros, Mathematical Programming 28, 1-14.

[14] Laan, G. van der, Talman, A.J.J., 1979. A restart algorithm for computing fixed points without an extra dimension, Mathematical Programming 17, 74-84.

[15] Laan, G. van der, Talman, A.J.J., 1981. A class of simplicial restart fixed point algorithms without an extra dimension, Mathematical Programming 20, 33-48.

[16] Laan, G. van der, Talman, A.J.J., Yang, Z., 2006. Solving discrete zero point problems, Mathematical Programming 108, 127-134.

[17] Laan, G. van der, Talman, A.J.J., Yang, Z., 2007a. A vector labeling method for solving discrete zero point and complementarity problems, SIAM Journal on Optimization 18, 290-308.

[18] Laan, G. van der, Talman, A.J.J., Yang, Z., 2007b. Computing integral solutions of complementarity problems, Discrete Optimization 4, 2385-2399.

[19] Merrill, O.H., 1972. Applications and Extensions of an Algorithm that Computes Fixed Points of Certain Upper Semi-Continuous Point-to-Set Mappings, PhD Thesis, University of Michigan, Ann Arbor.

[20] Murota, K., 2003. Discrete Convex Analysis, SIAM Publisher, Philadephia.

[21] Reiser, P.M., 1981. A modified integer labeling for complementarity algorithms, Mathematics of Operations Research 6, 129-139.

[22] Saigal, R., 1983. A homotopy for solving large, sparse and structured fixed point problems, Mathematics of Operations Research 8, 557-578.

[23] Scarf, H., 1967. The approximation of fixed points of a continuous mapping, SIAM Journal on Applied Mathematics 15, 1328-1343.

[24] Scarf, H., 1973. The Computation of Economic Equilibria, Yale University Press, New Haven. 
[25] Talman, A.J.J., Yang, Z., 2009. A discrete multivariate mean value theorem with applications, European Journal of Operational Research 192, 374-381.

[26] Tarski, A., 1955. A lattice-theoretical fixpoint theorem and its applications, Pacific Journal of Mathematics 5, 285-309.

[27] Todd, M.J., 1976. Computation of Fixed Points and Applications, Springer-Verlag, Berlin.

[28] Yang, Z., 2004. Discrete fixed point analysis and its applications, FBA Working Paper No. 210, Yokohama National University, Yokohama.

[29] Yang, Z., 2008. On the solutions of discrete nonlinear complementarity and related problems, Mathematics of Operations Research 33 (4), 976-990. 